\title{
PAHS AND ASTROBIOLOGY
}

\author{
L.J. Allamandola ${ }^{1}$
}

\begin{abstract}
In dense molecular clouds, the birthplace of stars and planets, interstellar atoms and molecules freeze onto extremely cold dust and ice particles. These ices are processed by ultraviolet light and cosmic rays forming hundreds of far more complex species, some of astrobiological interest. Eventually, these rain down on primordial planets where they take part in the young chemistry on these new worlds.

Although the IR spectroscopy and energetic processing of interstellar ice analogs have been studied for nearly 30 years, similar studies of PAH containing ices have only just begun. This paper presents recent results from laboratory studies on the vacuum ultraviolet (VUV) photochemistry of PAHs in water ice at low temperatures to assess the roles they play in the photochemical evolution of interstellar ices and their relevance to astrobiology. A number of "surprises" were found in these studies on PAH containing water-rich ices, indicating that PAHs likely play very important, unexpected roles in cosmic ice chemistry, physics and astrobiology.
\end{abstract}

\section{Introduction}

The story of PAHs and astrobiology is an important part of a complete revolution in our understanding of chemistry and biochemistry throughout the cosmos. Space was considered chemically barren for most of modern human history. That spell was broken some fifty years ago with the discovery of species such as $\mathrm{NH}_{3}$, $\mathrm{H}_{2} \mathrm{CO}, \mathrm{NH}_{2} \mathrm{CHO}$, and $\mathrm{CO}$ by radio astronomers during the late 1960's and early 1970's (Cheung et al. 1968; Snyder et al. 1969; Rubin et al. 1971; Wilson et al. 1970). Today there are almost 120 known interstellar species, the largest of which is $\mathrm{HC}_{11} \mathrm{~N}$. Add to this the discovery of the Unidentified Infrared (UIR) Emission Band spectra during the 1970's, the suggestion that the UIR bands originated in unbelievably large interstellar PAHs during the 1980's and the growing realization

1 NASA Ames Research Center, Space Science \& Astrobiology Division, MS 245-6, Moffett Field, CA 94035, USA 
over the last twenty years that PAHs are widespread and very abundant throughout much of the Cosmos and it is clear that we no longer simply see the Universe as an $\mathrm{H}$ dominated physicist's paradise.

While most of the work presented at this wonderful symposium has to do with using the emission from vibrationally excited, gas phase PAHs as probes of different environments, this paper discusses PAHs frozen in ices in dense molecular clouds because it is in these clouds that stars, planets and moons form. These PAHs are part of the extraterrestrial feedstock that contributes to the early chemistry and perhaps biochemistry on the primordial planets and moons.

In dense molecular clouds, atoms and molecules freeze onto the extremely cold dust and ice particles forming mixed molecular ices. Infrared spectroscopy of the clouds reveals the composition of these ices (e.g. van Dishoeck 2004). With the occasional exception of $\mathrm{CO}$, the abundance of the identified species is much higher in the ice than in the gas phase. Water is by far the major ice component, with frozen $\mathrm{CO}, \mathrm{CO}_{2}$, and $\mathrm{CH}_{3} \mathrm{OH}$ often, but not always, present at concentrations of roughly 10 to $20 \%$. The ices also contain $\mathrm{NH}_{3}, \mathrm{H}_{2} \mathrm{CO}$ and $\mathrm{CH}_{4}$ at about the few percent level. Within these clouds, and especially in the vicinity of star and planet forming regions, these ices are processed by ultraviolet light and cosmic rays forming hundreds of far more complex species, some of biogenic interest. This process is illustrated in Figure 1. Unidentified IR spectral structure indicates that other species are also present at the few percent level (e.g. Gibb \& Whittet 2002; Boogert et al. 2008). Some of these will likely be produced via in-situ UV photolysis of the interstellar ices. Although altering only a few percent of the ice, in-situ photochemistry favors chemical complexity and produces molecules and chemical groups within the ice that cannot be made via gas phase and gas-grain reactions. As these materials are the building blocks of comets and related to carbonaceous micrometeorites, they are likely to be important sources of complex organic materials delivered to habitable planets such as the primordial Earth. This chapter focuses on the unique roles that PAHs play in the chemical evolution of these cosmic materials and their relevance to astrobiology.

\section{Interstellar ice photochemistry and the production of biogenic molecules}

This section summarizes the photochemistry of mixed molecular and PAH containing interstellar ice analogs comprised of the most abundant interstellar ice species, with emphasis placed on the production of biogenically interesting molecules.

There is a large body of work on UV driven photochemistry in various low temperature (10-30 K) ice mixtures without PAHs (e.g. Allamandola et al. 1988; Bernstein et al. 1995; Greenberg et al. 1995; Gerakines et al. 1996, 2001). As these ices are warmed, the starting materials and most volatile photoproducts sublime, leaving non-volatile residues on the substrate. Mass spectroscopic analysis shows that hundreds of complex new species make up these residues (Greenberg et al. 2000; Dworkin et al. 2004). Of the overwhelming array of compounds produced 


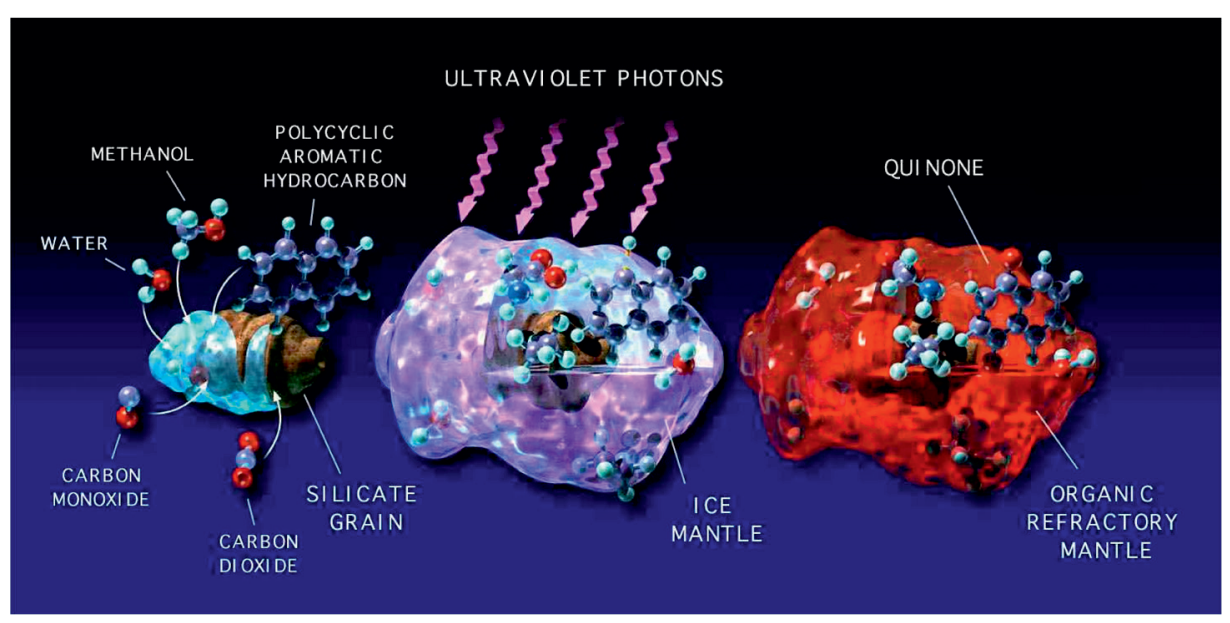

Fig. 1. The Greenberg model of interstellar ice mantle formation and chemical evolution. The mantle grows by condensation of gas phase species onto the cold dust grains. Simultaneously, surface reactions between these species, ultraviolet radiation and cosmic ray bombardment drive a complex chemistry. Figure reproduced from Bernstein et al. (1999a).

from even the simplest starting ice $\left(\mathrm{H}_{2} \mathrm{O}, \mathrm{CH}_{3} \mathrm{OH}, \mathrm{NH}_{3}\right.$, and $\left.\mathrm{CO}\right)$, only a few have been identified (Bernstein et al. 1995). In keeping with their expected low concentration and abundance with respect to the simpler ice species, clearcut IR spectroscopic evidence for these types of compounds in interstellar ices is presently lacking although, as mentioned above, weak spectral structure in the $5-8 \mu \mathrm{m}$ region of protostars is consistent with their presence (e.g. Gibb \& Whittet 2002; Boogert et al. 2008).

The residue is particularly important for astrobiology because it is quite plausible that this type of material is closely related to that preserved in comets, meteorites and interplanetary dust particles (IDPs), and it is believed that these sources deliver between 12 and 30 tons of organic material to Earth monthly (Love \& Brownlee 1993). During the period of great bombardment some 4 billion years ago, the amount of extraterrestrial organic material brought to the early Earth was many orders of magnitude greater. Thus, this organic residue could have played an important role in steering the early chemistry on habitable bodies such as the primordial Earth.

Motivated by the possibility that the residues may contain compounds that impact early Earth biochemistry, experiments have been directed to searching for classes of biogenically important species. Remarkably, although all of the starting compounds are water soluble, as shown in Figure 2, non-soluble droplets spontaneously form when the residues are added to liquid water (Allamandola et al. 1997; Dworkin et al. 2001). Droplet formation shows that some of the complex organic compounds produced by UV in these interstellar ice analogs are 


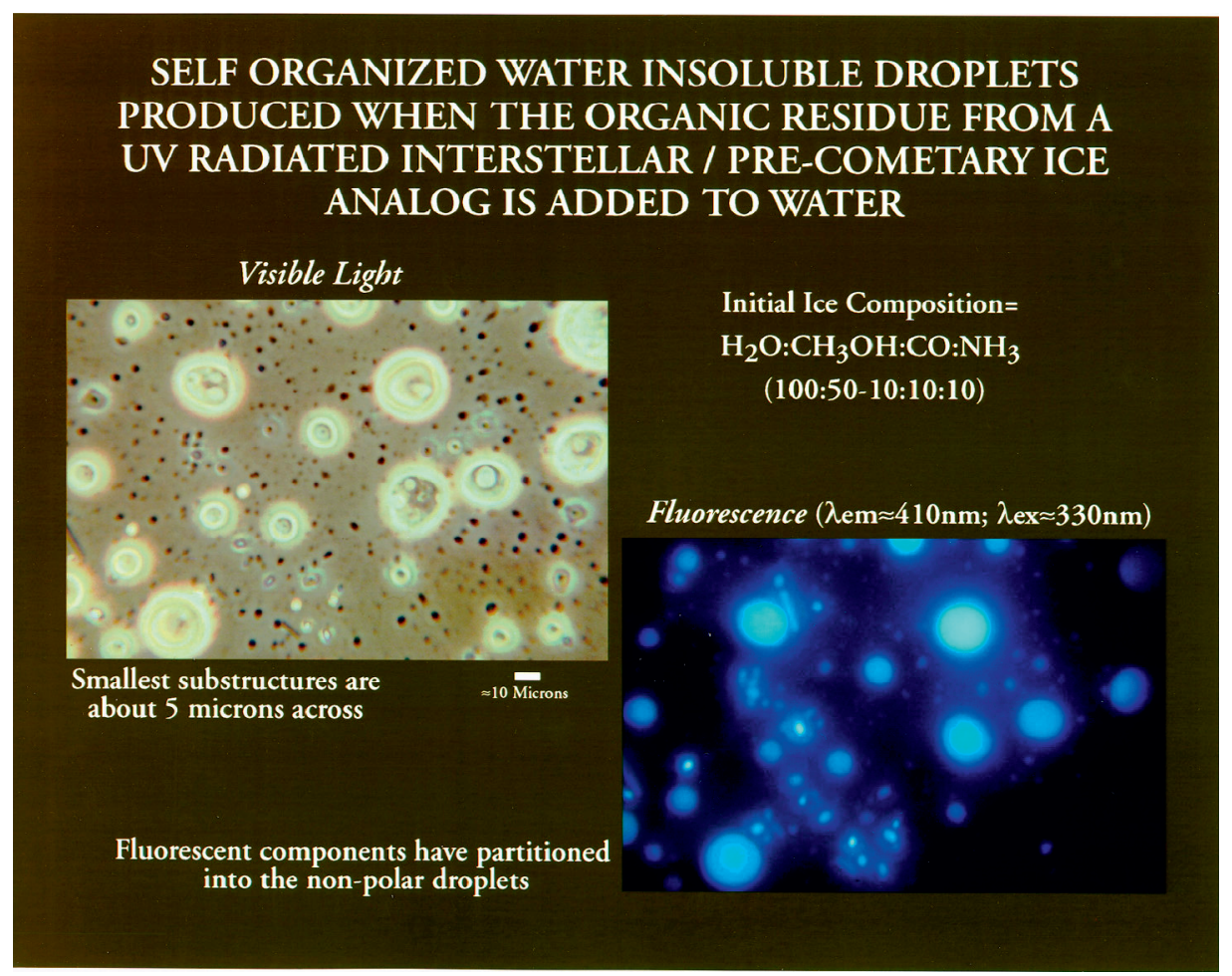

Fig. 2. Water insoluble, fluorescent droplets produced from the residue when it is added to water. The two images are of the same field.

amphiphilic, i.e. they have both a polar and non-polar component. These are the types of molecules that make up cell membranes, and membrane production is considered a critical step in the origin of life. These droplets are true vesicles (hollow droplets) with their interiors separated from the surroundings by their lipid multilayer. Vesicle formation is thought critical to the origin of life since vesicles provide an environment in which life can evolve, isolating and protecting the process from the surrounding medium (Deamer et al. 2002).

Figure 2 also shows that the membranes trap photoluminescent molecules that are also formed within the ice by UV irradiation (Allamandola et al. 1997; Dworkin et al. 2001). Thus, not only are vesicle forming compounds produced from the simplest and most abundant interstellar starting materials, complex organics which absorb low energy UV are also made. The ability to form and trap energy receptors within these structures is considered another critical step in the origin of life as it provides the means to harvest energy available outside the system. Interestingly, the vesicles formed from photoproducts of UV irradiated interstellar ice analogs are remarkably similar to those produced by extracts from the Murchison meteorite (Dworkin et al. 2001; Deamer et al. 2002). 
Another class of potentially important organic molecules brought to the Earth by meteorites are amino acids, the molecular building blocks of proteins and enzymes. The amino acids in meteorites are extraterrestrial as demonstrated by their deuterium enrichments - the highest of any species measured in a meteorite (Pizzarello \& Huang 2005). Since laboratory photolysis experiments of plausible presolar ice mixtures also produce amino acids and closely related compounds (Bernstein et al. 2002; Muñoz Caro et al. 2002; Agarwal et al. 1985), presolar ice photochemistry may be a source of these extraterrestrial amino acids.

As with other polyatomic interstellar species, PAHs should also become part of the ices in dense clouds. Weak, broad absorption bands in molecular cloud spectra are consistent with PAHs frozen in ice mantles at the few percent level, however they suffer severe blending with the strong absorption bands of the major ice components (Keane et al. 2001; Bernstein et al. 2005; Sandford et al. 2004; Bouwman et al. 2011; Bouwman et al., elsewhere in this volume). The chemistry of PAHs in cosmic ice analogs has also been investigated to understand the roles PAHs might play in astrochemistry and astrobiology. Photolysis of interstellar ice analogs containing pure PAHs does not destroy the parent PAH, but decorates it with chemical side-groups formed from the photoproducts of the other ice constituents. For example, in water ice, oxidized products found in the residues include aromatic ethers, alcohols, and ketones with structures as shown in Figure 3 (Bernstein et al. 1999b). Hydrogen atom addition transforms some of the edge rings into cyclic aliphatic hydrocarbons, creating molecules with both aromatic and aliphatic character and decreasing the overall degree of aromaticity. Likewise, UV and proton irradiation of PAHs in $\mathrm{NH}_{3}, \mathrm{CH}_{4}, \mathrm{CH}_{3} \mathrm{OH}, \mathrm{HCN}$ and $\mathrm{CO}_{2}$ ices produces aromatic structures decorated with amino $\left(-\mathrm{NH}_{2}\right)$, alkyl $\left(-\mathrm{CH}_{3},-\mathrm{CH}_{2}\right)$, hydroxyl $(-\mathrm{OH})$, cyano $(-\mathrm{CN})$, and carboxy $(>\mathrm{CO})$ side groups, again species similar to the compounds found in meteorites (Bernstein et al. 2003, 2002, 2001). Most recently, photolysis of the nitrogenated single ring aromatic molecule, pyrimidine, in water ice produced the nucleobase, uracil (Nuevo et al. 2009). These changes open up an aromatic based biogenic chemistry that is not available to the parent PAH or in non-PAH containing ices. For example, compounds closely related to aromatic ketones are widely used in current living systems for electron transport across cell membranes (hardware) while nucleic acids provide the backbone for information coding in DNA and RNA (software).

From both the astrochemical and astrobiological perspective, the production of such an interesting array of aromatic based compounds warranted a deeper understanding of the processes that occur within the ice during the UV irradiation of PAHs in cosmic ice analogs. However, because of severe line blending, the "traditional" laboratory approach using IR spectroscopy to follow chemical change within irradiated ices cannot provide this information (Bernstein et al. 2005; Sandford et al. 2004; Bouwman et al. 2011; Bouwman et al., elsewhere in this volume). It is for this reason that optical studies have been undertaken to follow the stepwise reaction pathways and kinetics of PAHs in water ice and to determine important properties such as ionization efficiencies, onset temperature of 


\section{Photolysis of PAHs in Water Ice Produces Alkanes, Ketones, Ethers, and Alcohols.}

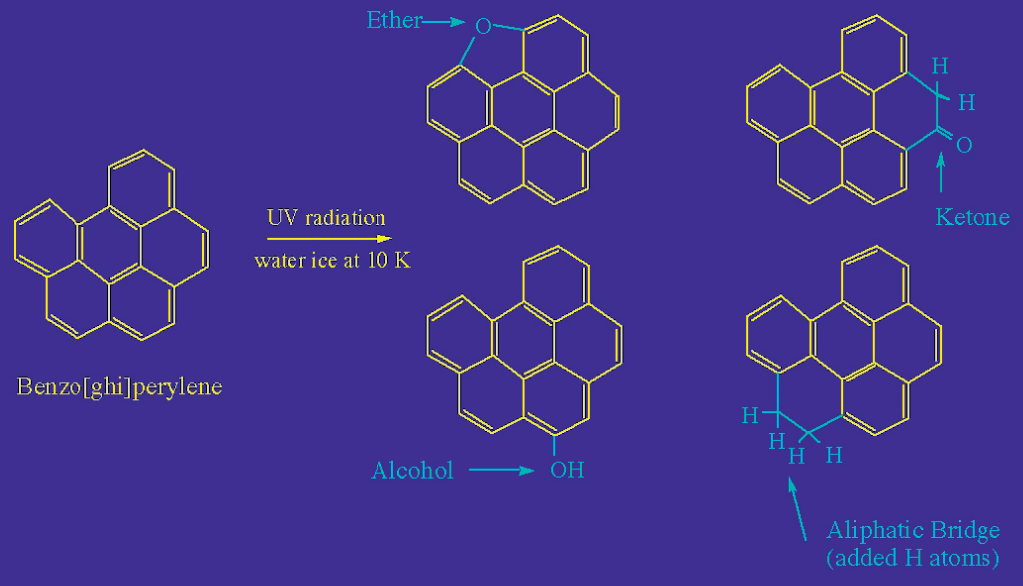

Both oxidation (alcohol, ether and ketone formation) and reduction (addition of hydrogen) reactions occur on photolysis of water ices containing PAHs. These are the same kinds of compounds observed in meteorites, fit spectra of emission objects and, in some cases, have biochemical significance.

Fig. 3. $\mathrm{PAH}$ structures produced by UV irradiation of $\mathrm{PAH}: \mathrm{H}_{2} \mathrm{O}$ ices at interstellar temperatures.

reaction during warm-up, possible identities of some of the reaction intermediates, and so on. The next section summarizes this work.

\section{Photochemistry and photophysics of PAHs in cosmic ice analogs}

A number of "surprises" were found in these studies on the photochemical processing of PAH containing water-rich ices. Foremost among these is that rapid photoionization is the dominant process that occurs when dilute, low temperature $\mathrm{PAH} / \mathrm{H}_{2} \mathrm{O}$ ices are initially exposed to UV radiation (Gudipati \& Allamandola 2003; Bouwman et al. 2010). As illustrated in Figure 4, a large fraction (generally 50-70\%) of the parent PAH is converted to $\mathrm{PAH}^{+}$within minutes of UV onset. If radiation is stopped at this point, the $\mathrm{PAH}$ cation appears to be stable indefinitely (on laboratory timescales) as long as the ice temperature is not raised. The PAH cation is also stabilized by the $\mathrm{H}_{2} \mathrm{O}$ ice to remarkably high temperatures. This is shown in Figure 5 which compares the spectrum of the quaterrylene (QTR, $\mathrm{C}_{40} \mathrm{H}_{20}$ ) cation in water ice at $20 \mathrm{~K}$ to that of the same sample at $120 \mathrm{~K}$ after a nearly one month, very slow, stepwise warm-up. The $\mathrm{QTR}^{+}$band only starts to weaken after the ice matrix is warmed above $120 \mathrm{~K}$, but the loss is slow up to 


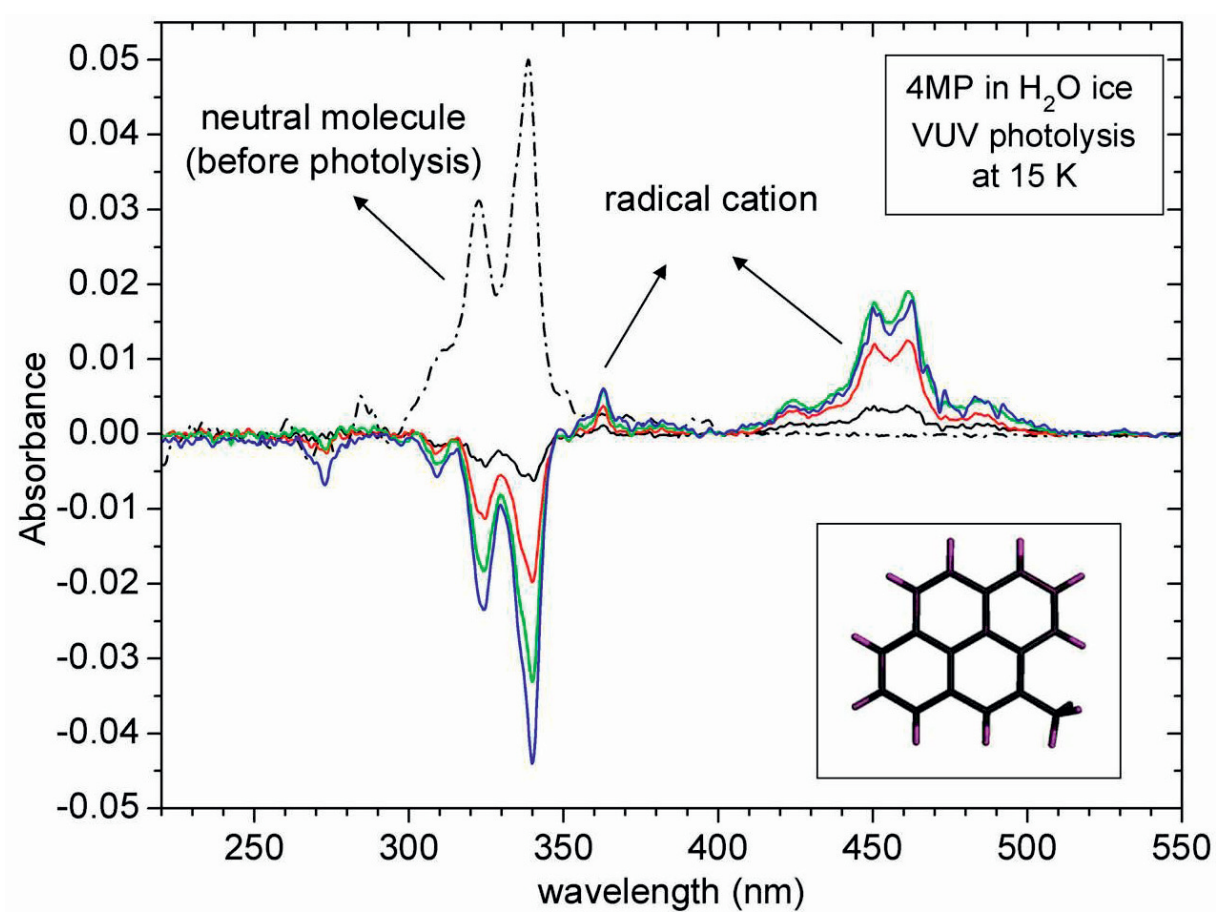

Fig. 4. Spectral evolution of $\mathrm{H}_{2} \mathrm{O} / 4$-methylpyrene (4MP, 1500/1) ice at $15 \mathrm{~K}$ as a function of VUV irradiation time showing the loss of neutral 4MP and simultaneous growth of $4 \mathrm{MP}^{+}$with VUV exposure. The dot-dashed line shows the absorbance spectrum before photolysis. The black, red, green, and blue solid lines show the loss of the neutral $4 \mathrm{MP}$ band and the simultaneous growth of $4 \mathrm{MP}^{+}$after $30,300,1200$, and $4800 \mathrm{~s}$, respectively, of in-situ VUV (vacuum UV) irradiation. The vibronic bands peaking near $340 \mathrm{~nm}$ correspond to neutral $4 \mathrm{MP}$, and those near $450 \mathrm{~nm}$ to the $4 \mathrm{MP}^{+}$photoproduct (Figure reproduced from Gudipati \& Allamandola 2003; see also Bouwman et al. 2010).

$\sim 140 \mathrm{~K}$. Although the loss increases at higher temperatures, band reduction stops when warm-up stops. Remarkably, the band is still evident at $170 \mathrm{~K}$, the temperature at which the ice sublimes (Gudipati \& Allamandola 2006). In addition, the ionization potential (IP) of PAHs trapped in water ice at low temperatures are at least $2 \mathrm{eV}$ lower than the corresponding gas phase IPs (Gudipati \& Allamandola 2004; Woon \& Park 2004)

These initial studies showed that PAHs likely play important but overlooked roles in cosmic ices, especially regarding the chemistry of charged species. A far more detailed understanding of the processes involved is necessary before one can incorporate these ideas into astrochemical models. To provide this critical information, Bouwman et al. $(2009,2010)$ and Bouwman et al. in press, have undertaken a systematic study of several PAHs in $\mathrm{H}_{2} \mathrm{O}$ ice, tracking the in-situ behavior of each PAH and their photoproducts within the ice itself as a function 


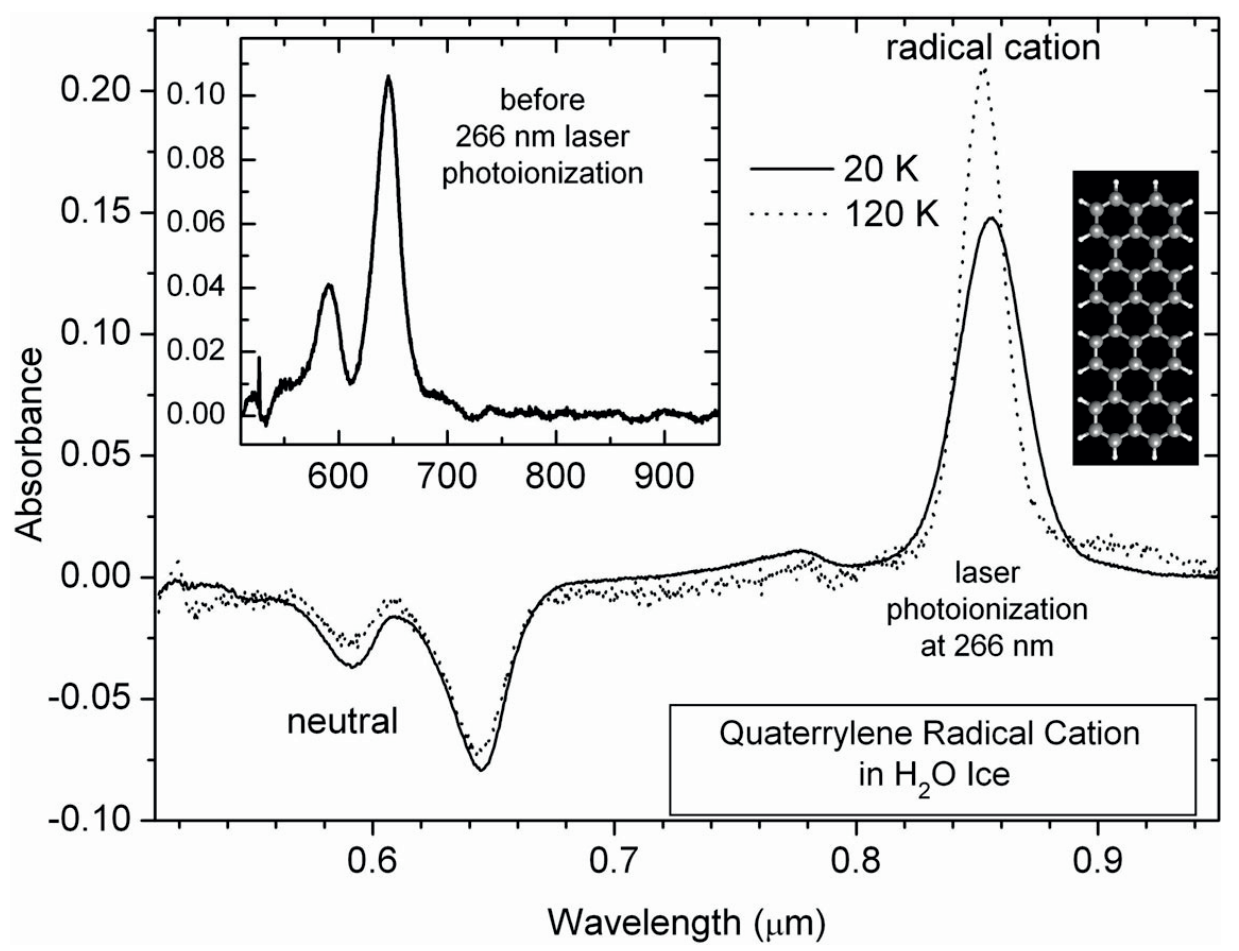

Fig. 5. The 520 to $940 \mathrm{~nm}(0.52$ to $0.94 \mu \mathrm{m})$ spectrum of quaterrylene (QTR, $\mathrm{C}_{40} \mathrm{H}_{20}$, upper right insert) in a water ice matrix. The spectrum of the freshly deposited QTR/ $\mathrm{H}_{2} \mathrm{O}$ $(\sim 1 / 500)$ ice at $20 \mathrm{~K}$ is shown in the upper left insert. The full spectrum (solid line) is that of the same ice after photolysis with $266 \mathrm{~nm}$ laser light. Negative bands represent depletion of the parent neutral QTR and positive bands represent generation of $\mathrm{QTR}^{+}$. The dotted-line spectrum was recorded at $120 \mathrm{~K}$, after step-wise warm-up over 28 days. The $\mathrm{QTR}^{+}$band areas are equal, showing $\mathrm{QTR}^{+}$is stable in water-ice at this temperature (Gudipati \& Allamandola 2006).

of ice temperature and UV irradiation time with sub-second responsivity. Figure 6 shows the dramatic effect ice temperature has on the photochemistry of the PAH pyrene $\left(\mathrm{Py}, \mathrm{C}_{16} \mathrm{H}_{12}\right)$ in water ice and Figure 2 in Bouwman et al., elsewhere in this volume, shows the evolution of each band as a function of photolysis time. The ability to measure spectra simultaneously with photolysis and with sub-second time resolution permits kinetic studies previously inaccessible and provides new insights into the processes occurring within the ice during photolysis (Bouwman et al. 2009, 2010; Bouwman et al. in press). Since these temperatures span the range of dust temperatures in dense clouds in the transition zones with star and planet forming regions and the ranges in the temperatures of icy Solar System bodies beyond the orbit of Jupiter, PAH driven ice photochemistry is likely to impact a variety of cosmic environments. 


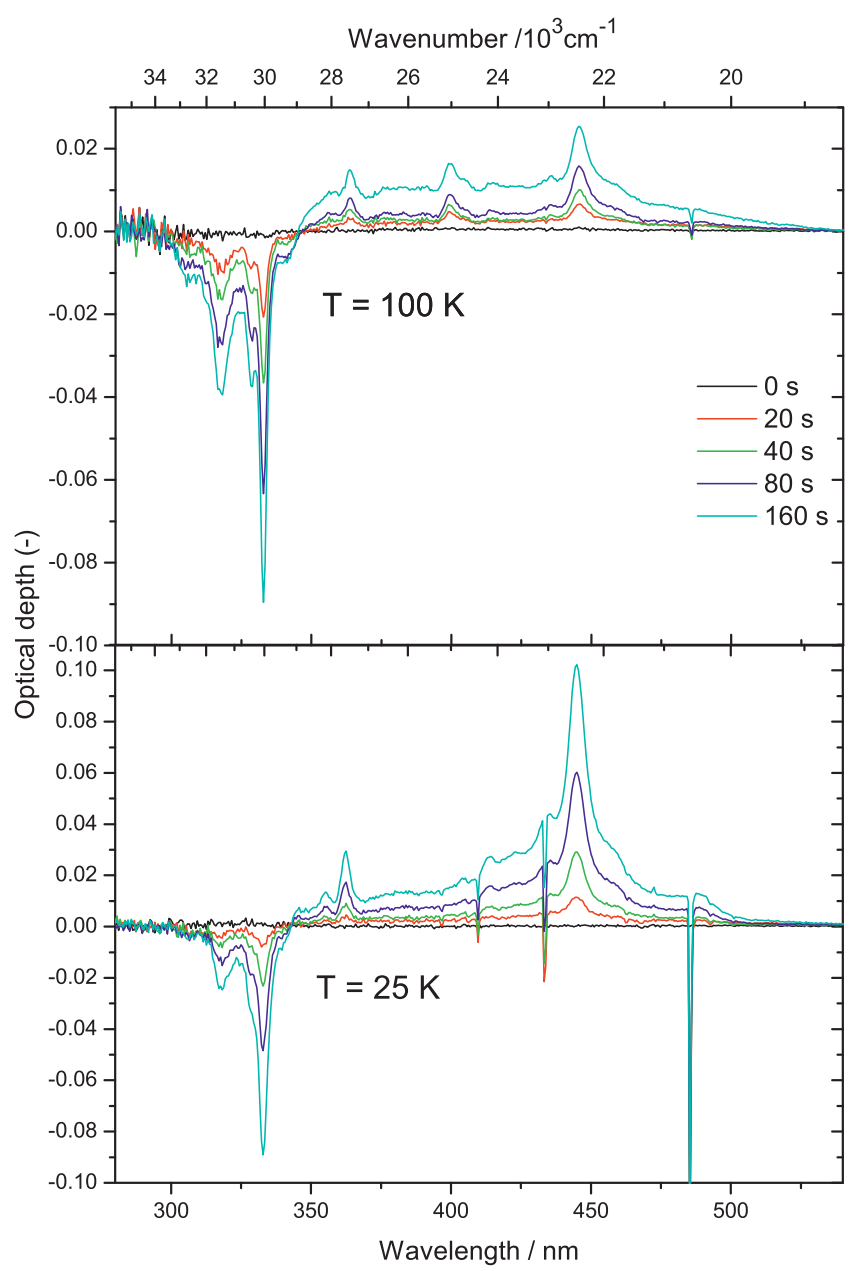

Fig. 6. The VUV induced spectroscopic changes in Pyrene: $\mathrm{H}_{2} \mathrm{O}$ ice at 25 (bottom) and $100 \mathrm{~K}$ (top) as a function of photolysis time. Temperature plays a critical role in determining photochemical pathways for PAH containing ice. The loss of the neutral pyrene $\left(\mathrm{Py}, \mathrm{C}_{16} \mathrm{H}_{12}\right)$ band complex near $330 \mathrm{~nm}$ is similar at both temperatures. However, in the $25 \mathrm{~K}$ ice, cation formation $(\sim 450 \mathrm{~nm})$ is favored over the range of species responsible for the broad, 350 to $500 \mathrm{~nm}$ absorption and the 400 and $405 \mathrm{~nm}$ band carriers. The opposite holds for the $100 \mathrm{~K}$ ice. Figure from Bouwman et al. (2010).

\section{Astrobiology}

The similarities between interstellar ice analog photoproducts with the extraterrestrial materials delivered to the Earth strengthens the case for taking interstellar PAHs and ices into account when pondering the exogenous contribution to the origin of life that is illustrated in Figure 7. 


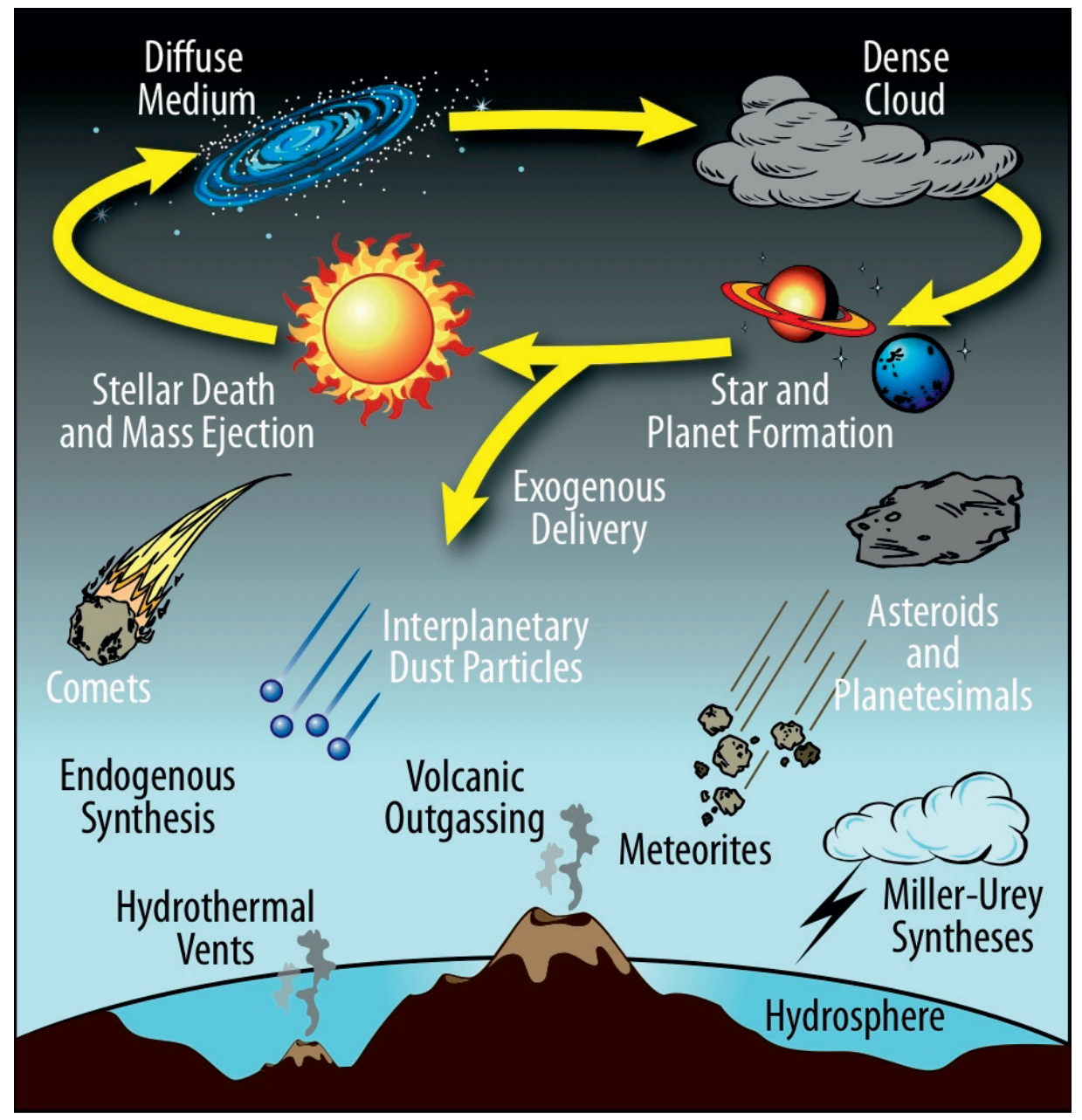

Fig. 7. The cycle of interstellar material that leads to the production and evolution of complex organic compounds that can be delivered intact to, and mixed with, those produced on planets and moons. There they participate in the primordial chemistry on these young objects. Figure courtesy Jason Dworkin, NASA Goddard Space Flight Center, adapted from Deamer et al. (2002).

Three possible roles that these interstellar materials might have played in the origin of life on Earth are:

(1) Supplier of basic, prebiotic raw materials from which biotic compounds were eventually produced on Earth. Comets, IDPs and meteorites were very likely responsible for raining a rich inventory of prebiotic molecules down onto the early Earth. Recall the variety of species that make up the bulk of interstellar ices. 
(2) Source of complex prebiotic materials poised to play a direct role once in a favorable environment. The work described here supports the idea that meteorites, dust, and comets could deliver species which are sufficiently complex to play a direct role in the chemistry of the origin of life. From this perspective, comets might represent quite potent prebiotic cocktails. All the complex species discussed above are produced by the irradiation of simple ice mixtures that are widespread throughout dense molecular clouds. Thus, it seems reasonable to hypothesize that wherever a planet or satellite is formed in the habitable zone, it will accrete these chemical remnants from the nascent cloud from infalling IDPs, cometary (formerly interstellar) and meteoritic materials. One of the challenges before us is to understand this chemistry and to assess the relative importance of this input into the origin of life versus an origin from the compounds produced on, or already present on, the primordial planet.

(3) Fountainhead of species undergoing the basic processes of life. Going one step further, this article ends on a speculative note. The results summarized here are quite startling when viewed from the perspective held not too long ago that chemistry in space was very limited. We have just started to scratch the surface of this complex chemistry, we know there are hundreds of residue compounds not yet analyzed and we have just realized that ion-moderated processes also play a role in ice chemistry. Given our level of ignorance at this stage, an intriguing possibility is the production, within the processed mixed molecular ices themselves - whether in the interstellar medium or a comet - of species poised to take part in the life process, or perhaps even at the earliest stages of what could be perceived of as rudimentary reactions of a living system.

This "jump-starting" of the life process by the introduction of such marginally biologically active species into the "warm pond" on a habitable planet may not be as far-fetched as it would at first seem. Although one could hardly imagine a better deep freeze than isolation within a comet, with temperatures measured in tens of degrees K, there are repeated episodes of warming for periodic comets. Since these warming episodes, which can be repeated many times in a given comet, can last anywhere from periods of weeks to several months, there is ample time for a very rich mixture of complex organics to develop even though these warming episodes are sandwiched between long periods of extreme cold. At this early stage in our understanding of cometary ices it already appears that comets contain many of the types of compounds which are considered important players in the origin of life, compounds which play central physical roles and compounds which play key chemical roles. Indeed there is evidence for nearly every class of organic chemical thought important in the "RNA world" in these irradiated ices and they are all in close proximity to one another. We are just beginning to appreciate the chemical complexity and possibilities of these ices. Thus, it is no longer inconceivable, at least to this author, that comets played a far more active role in the origin of life than simply that of a spectator-delivery system of raw materials. Perhaps Darwin's "warm little pond" is a warmed comet.

Summing up, in cold molecular clouds, the birthplace of planets and stars, interstellar molecules freeze into ice particles comprised of water, methanol, ammonia, 
carbon monoxide, carbon dioxide and PAHs. In these clouds, especially close to star and planet forming regions, these ices are processed by UV light, cosmic rays, radical surface reactions, etc. forming far more complex species, many of biogenic interest. During star and planet formation, many of these compounds become incorporated in comets and meteorites which eventually seed primordial planets where they take part in the budding chemistry on these young worlds.

While I have been blessed to have worked with many outstanding experimentalists and theoreticians over the years, I particularly wish to thank three exceptionally gifted experimentalists who made this chapter possible: Murthy Gudipati, Jordy Bouwman, and Harold Linnartz. They shined the first light into the microscopic details of PAH/ice processing. I am especially grateful to Harold Linnartz and NASA for making it possible for me to spend six wonderful months working in Leiden once again and Christiaan Boersma who, under severe time pressure, helped LaTeX this article. I also acknowledge support from NASA's Long Term Space Astrophysics, Exobiology, and Astrobiology programs for their continued support throughout this field's blossoming.

\section{References}

Agarwal, V.K., Schutte, W., Greenberg, J.M., Ferris, J.P., et al., 1985, Orig. Life, 16, 21 Allamandola, L.J., Sandford, S.A., \& Valero, G.J., 1988, Icarus, 76, 225

Allamandola, L.J., Bernstein, M.P., \& Sandlord, S.A., 1997, IAU Colloq. 161: Astron. Bioch. Orig. Search Life Universe, 23

Bernstein, M.P., Sandford, S.A., Allamandola, L.J., Chang, S., \& Scharberg, M.A., 1995, ApJ, 454, 327

Bernstein, M.P., Sandford, S.A., \& Allamandola, L.J., 1999a, Scient. Amer., 281, 26

Bernstein, M.P., Sandford, S.A., Allamandola, L.J., et al., 1999b, Science, 283, 1135

Bernstein, M.P., Dworkin, J.P., Sandford, S.A., \& Allamandola, L.J., 2001, Meteor. Planet. Sci., 36, 351

Bernstein, M.P., Dworkin, J.P., Sandford, S.A., Cooper, G.W., \& Allamandola, L.J., 2002, Nature, 416, 401

Bernstein, M.P., Elsila, J.E., Dworkin, J.P., et al., 2002, ApJ, 576, 1115

Bernstein, M.P., Moore, M.H., Elsila, J.E., et al., 2003, ApJ, 582, L25

Bernstein, M.P., Sandford, S.A., \& Allamandola, L.J., 2005, ApJS, 161, 53

Boogert, A.C.A., et al., 2008, ApJ, 678, 985

Bouwman, J., Paardekooper, D.M., Cuppen, H.M., Linnartz, H., \& Allamandola, L.J., 2009, ApJ, 700, 56

Bouwman, J., Cuppen, H.M., Bakker, A., Allamandola, L.J., \& Linnartz, H., 2010, A\&A, 511, A33

Bouwman, J., Mattioda, A.L., Linnartz, H., \& Allamandola, L.J., 2010, A\&A, 511, A33

Cheung, A.C., Rank, D.M., Townes, C.H., Thornton, D.D., \& Welch, W.J., 1968, Phys. Rev. Lett., 21, 1701

Deamer, D., Dworkin, J.P., Sandford, S.A., Bernstein, M.P., \& Allamandola, L.J., 2002, Astrobiology, 2, 371

Dworkin, J.P., Deamer, D.W., Sandford, S.A., \& Allamandola, L.J., 2001, Proc. Nat. Acad. Sci., 98, 815

Dworkin, J.P., Seb Gillette, J., Bernstein, M.P., et al., 2004, Adv. Space Res., 33, 67 
Gerakines, P.A., Schutte, W.A., \& Ehrenfreund, P., 1996, A\&A, 312, 289

Gerakines, P.A., Moore, M.H., \& Hudson, R.L., 2001, J. Geophys. Res., 106, 33381

Gibb, E.L., \& Whittet, D.C.B., 2002, ApJ, 566, L113

Greenberg, J.M., Li, A., Mendoza-Gomez, C.X., et al., 1995, ApJ, 455, L177

Greenberg, J.M., et al., 2000, ApJ, 531, L71

Gudipati, M.S., \& Allamandola, L.J., 2003, ApJ, 596, L195

Gudipati, M.S., \& Allamandola, L.J., 2004, ApJ, 615, L177

Gudipati, M.S., \& Allamandola, L.J., 2006, ApJ, 638, 286

Keane, J.V., Tielens, A.G.G.M., Boogert, A.C.A., Schutte, W.A., \& Whittet, D.C.B., 2001, A\&A, 376, 254

Love, S.G., \& Brownlee, D.E., 1993, Science, 262, 550

Muñoz Caro, G.M., et al., 2002, Nature, 416, 403

Nuevo, M., Milam, S.N., Sandford, S.A., Elsila, J.E., \& Dworkin, J.P., 2009, Astrobiology, 9,683

Pizzarello, S., \& Huang, Y., 2005, Geochim. Cosmochim. Acta, 69, 599

Rubin, R.H., Swenson, G.W., Jr., Benson, R.C., Tigelaar, H.L., \& Flygare, W.H., 1971, ApJ, 169, L39

Sandford, S.A., Bernstein, M.P., \& Allamandola, L.J., 2004, ApJ, 607, 346

Snyder, L.E., Buhl, D., Zuckerman, B., \& Palmer, P., 1969, Phys. Rev. Lett., 22, 679

van Dishoeck, E.F., 2004, ARA\&A, 42, 119

Wilson, R.W., Jefferts, K.B., \& Penzias, A.A., 1970, ApJ, 161, L43

Woon, D.E., \& Park, J.-Y., 2004, ApJ, 607, 342 
